DR. ARJUNE SEN (Orcid ID : 0000-0002-8948-4763)

DR. FÁBIO A. NASCIMENTO (Orcid ID : 0000-0002-7161-6385)

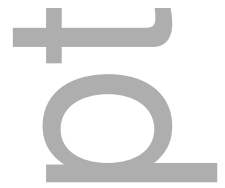

Article type : Full length original research paper

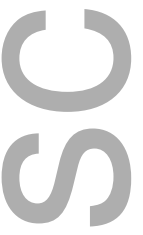

The phenotype of bilateral hippocampal sclerosis and its management in 'real life' clinical settings

Arjune Sen ${ }^{1 *+}$, Patricia Dugan ${ }^{2 *}$, Piero Perucca ${ }^{3,4^{*}}$, Daniel Costello ${ }^{5}$, Hyunmi Choi $^{6}$, Carl Bazil $^{6}$, Rod Radtke $^{7}$, Danielle Andrade ${ }^{8}$, Chantal Depondt ${ }^{9}$, Sinead Heavin ${ }^{10}$, Jane Adcock ${ }^{1}$, W Owen Pickrell ${ }^{11}$, Ronan McGinty ${ }^{1,5}$, Fabio Nascimento ${ }^{8}$, Philip Smith ${ }^{12}$, Mark I. Rees ${ }^{11}$, Patrick Kwan ${ }^{3,4,13}$, Terence J. $\mathrm{O}^{\prime}$ Brien $^{3,4}$, David Goldstein ${ }^{14}$, Norman Delanty ${ }^{10,15}$

${ }^{+}$Corresponding author:

Arjune Sen

Oxford Epilepsy Research Group

NIHR Biomedical Research Centre

Nuffield Department of Clinical Neurosciences

John Radcliffe Hospital,

Headington, Oxford,

OX3 9DU, UK

Telephone: +441865231891

Fax: + 441865234837

Email: arjune.sen@ndcn.ox.ac.uk

This is the author manuscript accepted for publication and has undergone full peer review but has not been through the copyediting, typesetting, pagination and proofreading process, which may lead to differences between this version and the Version of Record. Please cite this article as doi: $10.1111 /$ epi.14436

This article is protected by copyright. All rights reserved 
Running title: The phenotype of bilateral HS (29 characters)

Key words: Bilateral hippocampal sclerosis, epilepsy surgery, phenotype, seizure semiology, EPIGEN Number of text pages: 17 (main text)

Number of words (summary): 299

Number of words (main text): 3929

Number of tables (main and supplementary): 2 main and 4 supplementary

Number of figures (main and supplementary, and how many are in color): 4 main and 2 supplementary, all colour

Supporting figures and tables are included

\section{Author Disclosures:}

The authors declare no conflicts of interest

Affiliations:
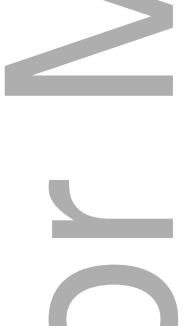

1. Oxford Epilepsy Research Group, NIHR Biomedical Research Centre, Nuffield Department of Clinical Neuroscience, John Radcliffe Hospital, Oxford. United Kingdom

2. Department of Neurology, New York University Langone Medical Center, New York, New York, U.S.A.

3. The Departments of Medicine and Neurology, The Royal Melbourne Hospital, The University of Melbourne, Parkville, Victoria, Australia.

This article is protected by copyright. All rights reserved 
4. The Departments of Neuroscience and Neurology, The Central Clinical School, Alfred Hospital, Monash University, Melbourne, Australia.

5. Department of Neurology, Cork University Hospital, Wilton, Cork. Ireland

6. Comprehensive Epilepsy Centre, Neurological Institute of New York, 710 West 168th Street, New York, USA

7. Department of Neurology, Duke University School of Medicine. Durham, NC, USA

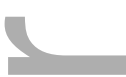

8. Toronto Western Hospital, 5W-445, 399 Bathurst Street, Toronto, Ontario. Canada

9. Department of Neurology, Hôpital Erasme - ULB, Cliniques universitaires de Bruxelles, Route de Lennik 808 - B - 1070 Bruxelles. Belgium

10. FutureNeuro Research Centre, Royal College of Surgeons in Ireland, Dublin, Ireland

11. Neurology Research Group, Institute of Life Science, Swansea University Medical School, Swansea, UK.

12. Department of Neurology, University of Wales, Cardiff, United Kingdom

13. Department of Medicine and Therapeutics, The Chinese University of Hong Kong, Hong Kong, China

14. Institute for Genomic Medicine, Columbia University Medical Center, Hammer Health Sciences 1408, 701 West 168th Street, New York, NY 10032

15. Department of Neurology, Beaumont Hospital, Dublin, Ireland

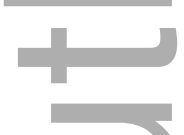

*: Joint first authors

The phenotype of bilateral hippocampal sclerosis and its management in 'real life' clinical settings

Abstract:

Objective:

This article is protected by copyright. All rights reserved 
There is little detailed phenotypic characterisation of bilateral hippocampal sclerosis (HS). We therefore conducted a multicentre review of all people with pharmacoresistant epilepsy and bilateral HS to better determine their clinical characteristics.

\section{Methods:}

Databases from 11 EPIGEN centres were searched. For identified cases, clinicians reviewed the medical notes, imaging, EEG, video-EEG and neuropsychometric data. Data were irretrievably anonymised and a single database populated to capture all phenotypic information. These data were compared with phenotyped cases of unilateral HS from the same centres

\section{Results:}

In total, 96 patients with pharmacoresistant epilepsy and bilateral HS were identified ( 43 female, 53 male; age range $8-80$ years). $25 \%$ had experienced febrile convulsions and $27 \%$ patients had experienced status epilepticus. The mean number of previously tried anti-epileptic drugs was 5.32 and the average number of currently prescribed medications was 2.99. There was a high incidence of eognitive and psychological difficulties $44.8 \%$ of patients had cognitive difficulties and $47.9 \%$ had psychiatric co-morbidity. 35.4\% (34/96) of patients continued with long-term medical therapy alone, another four being seizure free on medication. Sixteen patients proceeded to, or were awaiting, neurostimulation and eleven underwent surgical resection. One patient was rendered seizure-free post-resection with an improvement in seizures for three other cases.

By comparison, of 201 patients with unilateral HS a significantly higher number (44.3\%) had febrile convulsions and only $11.4 \%$ had experienced status epilepticus. Importantly, $41.8 \%$ (84/201) of patients with unilateral HS had focal aware seizures while such seizures were less frequently observed in people with bilateral HS, and were never observed exclusively ( $p=0.002$; Fisher's exact test)

\section{Significance:}

The current work describes the phenotypic spectrum of people with pharmacoresistant epilepsy and bilateral HS, highlights salient clinical differences from patients with unilateral HS and provides a large platform from which to develop further studies, both epidemiological and genomic, to better understand etiopathogeneisis and optimal treatment regimes in this condition.

Key words: Bilateral hippocampal sclerosis, epilepsy surgery, phenotype, seizure semiology

\section{Key points:}


1. Bilateral HS is a relatively rare, but important, cause of pharmacoresistant epilepsy

2. $25 \%$ of people with bilateral HS experienced a febrile seizure and no patient had focal aware seizures alone

3. $27 \%$ of patients with bilateral HS had experienced status epilepticus, not necessarily at the onset of seizures

4. Patients with bilateral HS had often tried many anti-epileptic medications and had high rates of cognitive and psychiatric co-morbidity

5. In this multicentre study, only a minority of patients with bilateral HS proceeded to surgical (palliative) resection of one hippocampus

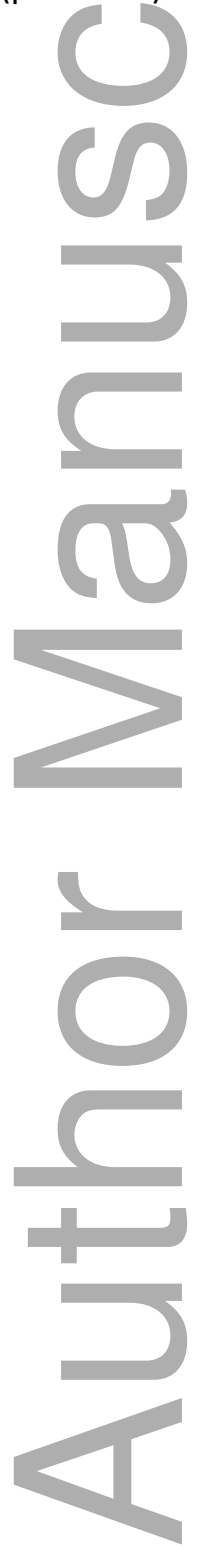




\section{The phenotype of bilateral hippocampal sclerosis and its management in 'real life' clinical settings}

1 1

\section{Introduction:}

The most common identifiable cause of pharmacoresistant focal epilepsy in adults is hippocampal sclerosis (HS). In unilateral HS, surgical resection of the affected hippocampus can associate with seizure freedom in up to $70 \%$ of cases ${ }^{1,2}$. However, it has long been appreciated that there are cases of drug-resistant focal epilepsy in whom HS is bilateral. For example, in 1958 Wilder Penfield identified two cases who developed profound memory difficulties following standard left temporal lobectomy. He hypothesised that these two patients had significant, but unsuspected, right hippocampal pathology such that when he resected the left hippocampus the effect was similar to bilateral temporal lobectomy ${ }^{3}$. Penfield's theory was substantiated many years later when one of his cases (patient PB) was analysed at autopsy and was found to have right sided hippocampal atrophy ${ }^{4}$. Penfield's noble publication, in which he illustrated unforeseen complications of resective surgery, led Scoville to contact him and highlight the case of Henri Molaison (Patient HM) who had undergone bilateral temporal lobectomies and subsequently developed dense anterograde amnesia. Milner travelled to Connecticut to study HM and it quickly became evident that bilateral temporal lobectomy was contraindicated owing to the profound cognitive sequela $e^{5,6}$.

Such observations, now six decades old, emphasize that bilateral hippocampal pathology can pose many difficulties. The development of MRI has enabled much better detection of bilateral $\mathrm{HS}^{7}, \mathrm{a}$ condition in which seizures are perceived to be very drug resistant and surgical options seem limited. The exact incidence of bilateral HS is unknown and there is little work specifically examining the phenotypic characteristics of patients with bilateral HS, particularly in patients with bilateral HS who do not proceed to surgical treatment. To address this, we evaluated the characteristics and management of patients with bilateral HS across a multicentre consortium (EPIGEN) and compared our data to a large collection of unilateral HS patients collated from the same centres

\section{Methods:}

This article is protected by copyright. All rights reserved 
EPIGEN is an international consortium of tertiary referral epilepsy centres which aims to deliver clinically-facing genomic research. All full member institutions of EPIGEN fColumbia University Aedical Center and New York University Langone Medical Center, New York, USA; Beaumont Hospital/Royal College of Surgeons of Ireland, Ireland; Duke University, Durham, USA; The Royal Melbourne Hospital, Australia; The Chinese University of Hong Kong, Hong Kong; Cork University Hospital, Ireland; Toronto Western Hospital, Canada; Swansea University Medical School, UK; Hôpital Erasme-ULB, Belgium; John Radeliffe Hospital/University of Oxford, UK), see supporting

Table 1 for details) searched clinically held databases for patients with bilateral HS. Inclusion criteria were kept deliberately broad (Supporting Table 2), with the principal criterion being that patients had to have evidence of bilateral HS on an epilepsy protocol brain MRI, as determined by a neuroradiologist and/or epileptologist. Bilateral HS was defined as demonstration of significant atrophy of both hippocampi with or without increase in signal of one or both hippocampi. Male and female patients of any age were included as were patients with dual pathology detected on brain imaging. We also specified that patients had to have tried two or more appropriate anti-epileptic drugs (AEDs) to enable study of patients with bilateral HS and pharmacoresistant epilepsy.

Detailed phenotypic analysis was performed through thorough review of medical notes/clinical databases including imaging reports, electroencephalogram (EEG)/video-EEG recordings, neuropsychometric assessments, operation notes, histology reports and clinical outcomes. Data were entered based on the medical information held at the EPIGEN centre and collected to the point at which medical documentation at the EPIGEN centre ceased.

Phenotypic data were de-identified and entered into a database with 25 principal entry fields (see Supporting Table $\mathbf{3}$ ). Researchers were encouraged to leave fields blank if there was any uncertainty. All data were pooled and two of the authors (AS and PD) analyzed the data. Any disagreements in data interpretation were resolved through careful discussion. Qualitative evaluation and relevant quantitative analyses were performed. We also collected data from a large collection of patients with unilateral HS attending the same EPIGEN centres to allow comparison of phenotypic characteristics between people with bilateral and unilateral HS. tohort of patients with unilateral HS collated from the same EPIGEN centres.

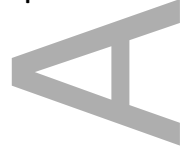

Results:

Basic phenotypic data

This article is protected by copyright. All rights reserved 
Nine centers identified patients from databases (range: 116 to 5000 patients) and two centers identified patients from clinics (average 1500-2000 patients per year). 96 patients with bilateral HS who met the inclusion criteria were studied ( 43 female, 53 male). The ethnic diversity of included patients reflects the centres that contribute to EPIGEN. The majority were Caucasian (72/96), 9 were of Asian descent ( 3 Indian, 4 Chinese, 2 not further specified) and 4 of African heritage. Racial origin was not documented formally in 11 cases.

The duration of follow-up at the EPIGEN centres ranged from 4 weeks to 58 years (mean 7.5 years). The age at time of data capture ranged from 8 to 80 years (mean 49.2 years; SEM 1.64) and age of seizure onset ranged from 0.5 to 63 years (mean 17.4 years; SEM 1.51 years). The average documented duration of seizures was 30.4 years (range 1-79 years; SEM 1.82 years).

\section{Etiopathogenesis of epilepsy}

Of the 96 patients studied, 14 (14.5\%) had a confirmed family history of epilepsy. However, in only 4 (4.2\%) patients was epilepsy identified in first-degree relatives and in two of these the family member's epilepsy was acquired (post-traumatic epilepsy, brain tumor).

The majority of patients did not have a documented history of febrile seizures (63 no evidence of febrile seizures; 9 not documented). In the 24 (25.0\%) patients who did have febrile seizures, two had prolonged febrile seizures. Interestingly, 5 patients who had febrile seizures had a positive family history of epilepsy, although in one case the affected relative had an acquired cause for their epilepsy. Similarly, 4 patients who had febrile seizures had additional clearly defined etiologies for developing epilepsy ( 1 meningitis in childhood, 2 meningoencephalitis in adulthood and 1 had neurosurgery in childhood (burr holes) owing to presumed intracranial hemorrhage).

The most common identifiable cause for bilateral HS was infection, principally meningoenecephalitis (Figure 1). Four patients developed epilepsy secondary to birth trauma and 4 after traumatic brain injury outside of the neonatal period. Similarly, four patients developed bilateral HS following new onset refractory status epilepticus (NORSE). Three patients were found to have a potentially causal auto-antibody (one voltage gated potassium channel (VGKC) antibody, one anti-glutamic acid decarboxylase (GAD) antibody and one not further specified), and were therefore classified as autoimmune epilepsy. The proportion of cases tested for autoantibodies was low (12/94 cases; 2 patients no data available), likely reflecting that cases were acquired over decades and many patients had a diagnosis of bilateral HS long before antibody-mediated epilepsy was recognised or specific antibody testing available. In the majority of cases, 61/96 (63.5\%), no cause was identified. 


\section{Seizure type}

In averaging all cases where a seizure frequency per month was recorded or could be derived (e.g. investigator recorded seizure frequency per week), the mean seizure frequency was 10.9 seizures per month. Seizure type was analysed in detail (Figure 2). The majority of patients had either focal seizures with impaired awareness and focal to bilateral tonic clonic seizures (44/96; 45.8\%). Focal impaired awareness seizures alone occurred in 23/96 (23.9\%) and focal seizures with and without impairment of awareness and focal to bilateral tonic clonic seizures in 16/96 (16.7\%). Four patients were reported to have bilateral tonic clonic seizures alone. No patient had only focal aware seizures. Most patients did not experience status epilepticus either at outset or at any point (64/96;66.7\%). In total $26(27.1 \%)$ patients had a previous history of status epilepticus and in 6 cases this information was not recorded.

\section{Medication history}

Patients included in this cohort had pharmacoresistant epilepsy and many patients had tried multiple AEDs. Only 7 cases did not have full datasets available for previously trialled medication and data were complete for currently prescribed treatments. The mean number of AEDs previously tried was 5.32 (range 1-15). The average number of currently prescribed AEDs was 2.99 (range 1-6).

The most commonly tried AED was carbamazepine followed by, in descending order, valproate, lamotrigine, phenytoin and levetiracetam (Figure 3A). By contrast, levetiracetam was the most commonly prescribed current AED followed, again in descending order, by lamotrigine, clobazam, valproate and carbamazepine (Figure 3B). Various other second and third line AEDs were also either tried previously or were being prescribed as a current medication. In a small number of cases intravenous immunoglobulin was given owing to a putative autoimmune aetiology. Steroids may also have been prescribed for these patients and possibly plasma exchange. However, no definitive comments can be made on immunomodulation administered to patients with bilateral HS as most centres did not document medications beyond conventional AEDs.

\section{Co-morbidities}

Many patients with bilateral HS had not undergone formal neuropsychometric testing with neuropsychometric scores only rarely given. Almost half of the patients were reported to have some cognitive impairment. In 17/96 (17.7\%) memory impairment was identified while an additional $26 / 96$ or $27.1 \%$ were reported to have cognitive difficulties ( 21 intellectual difficulties and 5 non- 
specified cognitive impairment). One patient was reported to have learning difficulties, 3/96 (3.1\%) had developmental delay and 1 patient had attention deficit hyperactivity disorder (Supporting Figure $1 \mathrm{~A})$. In 42/96 cases (43.8\%) there was no evidence for cognitive impairment and in 6 (6.3\%) cases insufficient information was recorded.

Psychiatric co-morbidity (Supporting Figure 1B) affected almost half of the patients (46/96; 47.9\%), with particular representation of depression, anxiety or both. A small number (4/96; 4.2\%) developed inter-ictal psychosis with 4 patients having experienced post-ictal psychosis. Three patients had dissociative seizures and $2.1 \%(2 / 96)$ had misused recreational substances.

These patients also experienced many other medical co-morbidities, particularly osteoporosis (6/96; $6.3 \%)$ and headache $(5 / 96 ; 5.2 \%$ - data not shown).

\section{Investigations}

Only patients with radiologically-confirmed bilateral HS were included. MRI also revealed additional cavernomas in 3 (3.5\%) patients, post-traumatic change in two (2.4\%) and cortical dysplasia in one.

Most patients (85/96; 88.5\%) were admitted for video-telemetry with a wide range of both interictal and ictal EEG findings (Supporting Figure 2A, B). Thirty-six (37.5\%) had bi-temporal interictal discharges with $14 / 36(38.9 \%)$ reporting no cognitive impairments while 22/36 (61.1\%) had cognitive impairment/intellectual disability/learning disability, or memory impairment. One quarter of cases $(24 / 96 ; 25.0 \%)$ had bilateral onset to seizures and a majority of this group $(18 / 24 ; 75.0 \%)$ had cognitive difficulties.

Eleven (11.5\%) patients underwent intracranial EEG recordings and, of these, seizures had unilateral temporal onset in 5 (1 right, 4 left), bi-temporal onset in 4 , unilateral hemispheric onset in 1 , and multifocal onset in 1 (Supporting Table 4). Only 17 (17.7\%) patients underwent Wada (sodium amobarbital) testing, perhaps as many had already been determined to not be a candidate for surgical resection. While difficult to interpret in isolation, Wada testing did demonstrate left hemispheric dominance in most cases.

\section{Surgical interventions}

A total of $18(18.75 \%)$ patients underwent epilepsy surgery - intracranial recording and/or resection. Of the eleven patients who underwent intracranial EEG monitoring, 4 subsequently had a resection (anterior temporal lobectomy, selective amygdalohippocampectomy or corticectomy). Seven patients had single stage resections (Supporting Table 4). In 8 of the temporal lobectomies, HS was 
confirmed on pathological analysis with no histopathological data available for the other 2 cases. In the patient who underwent corticectomy, non-specific findings were demonstrated.

\section{Outcomes}

Outcome to the point of the most recent appointment in the EPIGEN centre was recorded (Figure 4). In $23(24.0 \%)$ cases outcome data were not recorded and one patient died. Four patients were seizure free on anti-epileptic medication alone (4/96; 4.2\%). Of the 11 patients who underwent surgical resection, only 1 patient was rendered completely seizure free, and 3 (27.3\% of patients who had undergone resection) showed improvement in seizure frequency.

Around a third of patients continued with medical management alone (34/96, 35.4\%). A proportion of patients underwent, or are considering, neurostimulation therapy with either vagal nerve stimulation (9/96; $9.4 \%)$, responsive neurostimulation $(6 / 96 ; 6.3 \%)$ or, in one case, both.

\section{Comparison of people with bilateral HS to those with unilateral HS}

We specifically wished to delineate how specific certain findings were to bilateral HS. We therefore compared our 96 patients with 201 cases of unilateral HS from EPIGEN Centres. Of these 201 cases, 199 had pharmacoresistant epilepsy, with two having on-going seizures despite an adequate trial of one anti-epileptic drug, and 154 had proceeded to surgical resection. Key comparative phenotypic data from the patients with unilateral HS are highlighted in Table 1. As illustrated, patients with unilateral HS were significantly more likely to have experienced febrile seizures ( $44.3 \%$ compared to $25 \%$ in bilateral HS; $p=0.0002$; Fisher's exact test) and were less likely to have experienced status epilepticus ( $11.4 \%$ in unilateral HS versus $27.1 \%$ in bilateral HS). Some caution must be applied to statistical analysis of status epilepticus as data were not provided for over half of patients with unilateral HS. Also, whereas focal aware seizures were a common seizure type in patients with unilateral $\mathrm{HS}$, being found in 84 cases $(41.8 \%)$, such seizures were less frequent in people with bilateral HS ( $p=0.002$; Fisher's exact test) and no patient with bilateral disease had only focal aware seizures.

\section{Discussion}

The current study is the largest phenotypic characterisation of bilateral HS and epilepsy to date. Importantly, the study collates data from multiple epilepsy centres thereby reducing bias and better reflecting 'real-life' clinical practice across multiple countries. Previous work ${ }^{8-11}$ is summarised in Table 2 coupled with a summary of our data. 
It is established that The contralateral hippocampus in patients with unilateral hippocampal sclerosis may have certain imaging changes ${ }^{12-15}$ or pathological features detected at post-mortem ${ }^{16}$.

Volumetry, particularly with appropriate normalisation to control values, enhances detection of bilateral hippocampal atrophy ${ }^{13,15,17}$ and in studies of patients with unilateral $\mathrm{HS}$, the contralateral hippocampus can be significantly smaller than control hippocampi although also larger than the hippocampus ipsilateral to the lobectomy ${ }^{15}$. MR techniques have been utilised to try and refine bilateral mesial temporal lobe epilepsy (mTLE) and, for example, predict surgical outcome in patients with bilateral hippocampal atrophy ${ }^{18}$. There are also some patients with bilateral HS who are drug responsive. Inclusion of drug responsive cases or those with hippocampal atrophy defined by volumetric MR analysis would have increased the number of people studied, but our aim was to better describe patients with pharmacoresistant epilepsy and bilateral HS visible on MR imaging in a standard clinical setting to determine whether detailed phenotypic analysis might offer insights to improve clinical care going forwards

Of the 96 patients we studied most had: 1) a very high number of seizures per month; 2) previous exposure to multiple AEDs and 3) were on average taking around three AEDs concurrently. The pattern of drug prescription likely mirrors trends in clinical practice. The of some patients had very long epilepsy histories and it is perhaps unsurprising that a majority of patients had been exposed to carbamazepine although this was not a common current prescription with levetiracetam and lamotrigine now being favoured.

Complex febrile seizures (either prolonged convulsion, convulsion associated with unilateral weakness or febrile status epilepticus in childhood) associate with an increased risk of subsequently developing epilepsy ${ }^{19,20}$. Some studies have reported an incidence of febrile seizures of nearly $50 \%$ in patients with unilateral $\mathrm{HS}^{21}$ and our data detected febrile seizures in $44.3 \%$ of unilateral $\mathrm{HS}$ cases. Similarly, previous work has shown that in patients with temporal lobe epilepsy and a background of febrile convulsions a majority will have unilateral hippocampal atrophy ${ }^{17}$. This does not seem to be recapitulated in bilateral HS.As in other work ${ }^{10}$ a relatively smaller proportion of our patients with bilateral HS had a history of febrile seizures (26\%) and only two cases in this cohort had prolonged febrile convulsions. Five patients with febrile seizures also had a family history of epilepsy although full information about family history of epilepsy may be lacking as the study

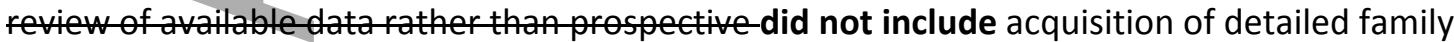
pedigrees. Previous genome-wide association studies have suggested a possible association of mTLE in patients who have previously had febrile convulsions and mutations in the SCN1A gene ${ }^{22}$. 
However, in the current cohort those who had a history of febrile seizures often also described other risk factors for developing bilateral HS, for example a subsequent meningoencephalitic illness.

While chronic epilepsy, even in patients with pre-existing hippocampal malformations, does not necessarily result in $\mathrm{HS}^{23}$, it is well recognised that prolonged status epilepticus can result in profound hippocampal volume loss and associate with subsequent pharmacoresistant epilepsy ${ }^{24}$. Although patients from the latter category are represented in the current cohort, for example the 3 patients in whom NORSE was recorded as the underlying aetiology, most patients did not have a history of status epilepticus. The most common aetiology was infection and other work has also demonstrated that bilateral hippocampal atrophy may be more likely after a meningitic or encephalitic illness ${ }^{17}$. A small number of patients were demonstrated to have a potentially causal antibody, but this proportion may be higher in future studies as more antibodies implicated in the pathogenesis of epilepsy are identified and testing for such antibodies increases.

Despite the large number of cases included here, it is not possible to define an exact aetiology for bilateral HS. There are features to suggest that bilateral HS is a different entity to unilateral HS, but it may be that bilateral hippocampal damage represents the final common pathway of a heterogeneous collection of rare epilepsy syndromes. Similar to the increasing recognition that antibody-mediated disease may contribute to refractory status epilepticus, it can be speculated that certain insults, perhaps particularly infection, may lead to development of bilateral HS in people with a genetic susceptibility to this condition

Intriguingly no patient with bilateral HS had only focal aware seizures and the incidence of focal aware seizures overall (i.e. even when considered in combination with other seizure types) was also significantly lower than in patients with unilateral HS. The exact reasons for this are uncertain. It might, for example, be that an individual seizure from a diseased hippocampus will likely cause impairment of awareness in the presence of an anatomically abnormal contralateral hippocampus.

As would be predicted in a group of patients with pharmacoresistant epilepsy, there were high rates of cognitive and psychological co-morbidity. Were all patients to have neuropsychometric testing, the incidence of cognitive impairment might be higher, particularly as people with unilateral HS may be below the $50^{\text {th }}$ centile in all cognitive domains ${ }^{25}$. There was also a high degree of psychopathology with over a third of patients reporting depression, anxiety or both. Again, one may predict higher rates of psychiatric symptomatology were this to be formally evaluated for all patients.

Only 11 (11.5\%) patients proceeded to resective surgery, which is low compared to other studies. In 1996, Arruda and colleagues evaluated 74 consecutive patients with mTLE undergoing surgical 
resection. When measured by volumetry, 17 of these cases had bilateral hippocampal atrophy and while the outcomes in this group were less favourable than in unilateral HS, 61.7\% had class I or II outcome according to Engel's modified classification ${ }^{26}$. More recently, Vanli-Yuvaz and colleagues reviewed 124 patients who had undergone epilepsy surgery for hippocampal sclerosis at their centre in Turkey ${ }^{11}$. In that study 93 patients had unilateral HS and 31 had bilateral HS. The authors report that $16.1 \%$ of bilateral HS were not pharmacoresistant and that six of nine patients with bilateral HS who underwent unilateral temporal lobectomy were rendered seizure free ${ }^{11}$. Similarly in 2013 Malter and colleagues evaluated patients at a single centre in Germany ${ }^{9}$. They also identified 31 cases of bilateral HS and eleven of these proceeded to surgery with resection of the putatively more epileptogenic hippocampus. Of those that did progress to surgery, seizure freedom rates at 12 and 24 months were similar to cases of unilateral HS and no patient with bilateral HS became globally amnestic post-resection. An older study evaluated 28 patients with bilateral independent temporal lobe seizures detected with intracranial recording of whom 15 proceeded to resection ${ }^{27}$. Ten patients were rendered seizure free and of those seven were shown to have unilateral HS on imaging or a lateralised Wada result. In the five who had persistent seizures, such structural or functional lateralisation was not evident ${ }^{27}$. In our study, only a single patient became seizure free post-resection.

The reasons for our relatively low percentage of operated cases may be multifactorial including patient choice, physician choice and increasing availability of other therapies, particularly new AEDs and neurostimulation. Additionally, in this cohort, very few patients $(17 / 96 ; 17.7 \%)$ had clear evidence of unilateral temporal lobe epilepsy on non-invasive video-telemetry while 24 patients (25\%) had evidence of bilateral temporal lobe seizures. This may in turn account for the lower number of patients proposed for invasive investigations (Wada testing: 17/96 (17.7\%); intracranial recording 11/96 (11.5\%)).

While the previously published surgical case series have reported good seizure outcomes ${ }^{9,11}$, unilateral resection in patients with bilateral HS, even if the patient is rendered seizure free, can compromise remaining memory function ${ }^{27,28}$. Moreover, there is now patients with bilateral mTLE can have independent seizure generation in each hippocampus on a

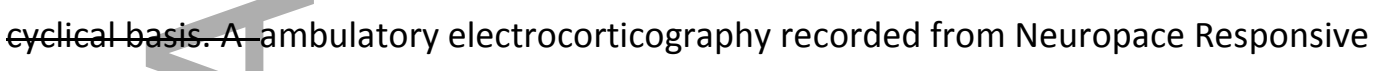
Neurostimulation devices has now shown that seizures may arise for several weeks in one hippocampus before then switching to the other ${ }^{29,30}$. Given that video-telemetry is typically of one to two weeks' duration, this may artificially skew interpretation if all, or most, of the seizures during EEG recording happen to arise from one of the sclerosed hippocampi. 
At a pathological level, those patients who underwent temporal lobectomy were found to have HS on histopathological examination, where this was available. Interesting work has shown that in postmortem cases of HS there can be bilateral accumulation of tau deposits within the sclerosed hippocampus ${ }^{31}$ and bilateral dentate granule cell dispersion ${ }^{16}$. The contributions that such bilateral pathology may make to cognitive difficulties and/or epileptogenesis, particularly in patients in whom bilateral HS is detected in vivo, would be worthy of further investigation.

The current work would suggest that proceeding to resection in patients with bilateral HS is not that common in real life practice and perhaps viewed more as a palliative, rather than a curative, procedure, even in specialist centres that are able offer the range of epilepsy surgeries available. Moreover, the presence or absence of focal aware seizures might be valuable in stratifying potential epilepsy surgical candidates going forwards. For example, the presence of focal aware seizures might be indicative of unilateral HS and, perhaps more importantly, the absence of focal aware seizures in a patient without obvious markers of bilateral HS might be indicative of bilateral disease and consequently a poorer post-operative prognosis. Careful delineation of seizure phenotype could therefore offer insights into the possibility of sub-clinical bilateral disease, just as Penfield was attuned to so many years ago.

\section{Limitations}

This study was limited to retrospective review of patient data held at the individual EPIGEN centres. Despite making data entry fields formulaic, there were inevitable subjective differences in the way individual investigators entered data. While objective information was provided for the majority/all patients for certain fields (for example seizure type and medications), more subjective data fields such as cognitive and psychological difficulties were less complete restricting interpretation.

Similarly, there are limitations in retrospectively comparing bilateral HS cases to patients with unilateral HS even if the cases are drawn from the same Epilepsy Centres - more patients with unilateral HS having had formal neuropsychometric and neuropsychiatric evaluations, for example. A well-defined multi-centre prospective study examining patients with bilateral HS and comparing such patients to prospectively phenotyped cases of unilateral HS would seem worthwhile.

\section{Conclusions}

This article is protected by copyright. All rights reserved 
Patients with pharmacoresistant epilepsy and bilateral HS can pose significant challenges to clinicians trying to ameliorate both seizures and associated co-morbidities. In the current study, which samples clinical practice across multiple centres in different countries, such patients are shown to: 1 ) have frequent seizures with no patient having focal aware seizures alone; 2 ) have a relatively low frequency of febrile seizures compared to unilateral HS; 3 ) often have no identified etiology to the bilateral HS and 4) do not often proceed to surgical resection. A prospective study examining a large series of cases with bilateral HS, building on data presented here, would help to better inform etiology as, for example, antibody testing becomes more established. Similarly, genomic work in a well phenotyped group of patients with bilateral HS might provide further distinctions from unilateral HS. Epidemiological work is also required to better determine whether a more aggressive or more circumspect approach is appropriate when deciding to offer unilateral hippocampectomy in patients with bilateral disease.

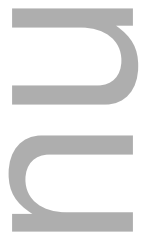

\section{Acknowledgements:}

The research was supported by the National Institute for Health Research (NIHR) Oxford Biomedical Research Centre (BRC)

We confirm that we have read the Journal's position on issues involved in ethical publication and affirm that this report is consistent with those guidelines.

None of the authors declare any conflicts of interest

\section{References:}

1. Wiebe S, Blume WT, Girvin JP, et al. A randomized, controlled trial of surgery for temporallobe epilepsy. N Engl J Med. 2001;345:311-8.

2. Spencer S, Huh L. Outcomes of epilepsy surgery in adults and children. Lancet Neurol. 2008;7:525-37.

3. Penfield W, Milner B. Memory deficit produced by bilateral lesions in the hippocampal zone. A M A Arch Neurol Psych. 1958;79:475-97. 
4. Squire LR. The Legacy of Patient H.M. for Neuroscience. Neuron. 2009; 61:6-9.

5. Scoville WB, Milner B. Loss of recent memory after bilateral hippocampal lesions. J Neuropsych Clin Neurosci. 1957;20:11-21.

6. Corkin S. What's new with the amnesic patient H.M.? Nat Rev Neurosci. 2002;3:153-60.

7. Jackson GD, Berkovic SF, Tress BM et al. Hippocampal sclerosis can be reliably detected by magnetic resonance imaging. Neurology. 1990;40:1869-75.

8. Ravat $S$, Rao $P$, lyer $V$, et al. Surgical outcomes with non-invasive presurgical evaluation in MRI determined bilateral mesial temporal sclerosis: A retrospective cohort study. Int J Surg. 2016; 36:429-435

9. Malter MP, Tschampa HJ, Helmstaedter $\mathrm{C}$ et al. Outcome after epilepsy surgery in patients with MRI features of bilateral ammon's horn sclerosis. Epilepsy Res. 2013;105:150-7.

10. Van Paesschen W, Connelly A, King MD et al. The spectrum of hippocampal sclerosis: A quantitative magnetic resonance imaging study. Ann Neurol. 1997;41:41-51.

11. Vanli-Yavuz EN, Baykan B, Sencer S et al. How Different Are the Patients With Bilateral Hippocampal Sclerosis From the Unilateral Ones Clinically? Clin EEG Neurosci. 2017;48:20916.

12. Koepp MJ, Labbé C, Richardson MP, et al. Regional hippocampal [11C]flumazenil PET in temporal lobe epilepsy with unilateral and bilateral hippocampal sclerosis. Brain. 1997;120:1865-76.

13. King D, Spencer SS, McCarthy G et al. Bilateral Hippocampal Atrophy in Medial Temporal Lobe Epilepsy. Epilepsia. 1995;36:905-10.

14. Hogan RE, Carne RP, Kilpatrick CJ et al. Hippocampal deformation mapping in MRI negative PET positive temporal lobe epilepsy. J Neurol Neurosurg Psychiatry. 2008;79:636-40.

15. Quigg $\mathrm{M}$, Bertram EH, Jackson T et al. Volumetric magnetic resonance imaging evidence of bilateral hippocampal atrophy in mesial temporal lobe epilepsy. Epilepsia. 1997;38:588-94.

16. Thom $\mathrm{M}$, Martinian $\mathrm{L}, \mathrm{Catarino} \mathrm{C}$ et al. Bilateral reorganization of the dentate gyrus in hippocampal sclerosis: A postmortem study. Neurology. 2009;73:1033-40.

17. Free SL, Li LM, Fish DR et al. Bilateral hippocampal volume loss in patients with a history of 
encephalitis or meningitis. Epilepsia. 1996;37:400-5.

18. Li LM, Cendes F, Antel SB et al. Prognostic value of proton magnetic resonance spectroscopic imaging for surgical outcome in patients with intractable temporal lobe epilepsy and bilateral hippocampal atrophy. Ann Neurol. 2000;47:195-200.

19. Annegers JF, Hauser WA, Shirts SB et al. Factors prognostic of unprovoked seizures after febrile convulsions. N Engl J Med. 1987;316:493-8.

20. Shinnar S. Febrile Seizures and Mesial Temporal Sclerosis. Epilepsy Curr. 2003;3:115-8.

21. Bower SP, Kilpatrick CJ, Vogrin ST et al. Degree of hippocampal atrophy is not related to a history of febrile seizures in patients with proved hippocampal sclerosis. J Neurol Neurosurg Psychiatry. 2000;69:733-8.

22. Kasperaviute D, Catarino CB, Matarin M, et al. Epilepsy, hippocampal sclerosis and febrile seizures linked by common genetic variation around SCN1A. Brain. 2013;136:3140-50.

23. Sen A, Thom M, Martinian L et al. Hippocampal malformations do not necessarily evolve into hippocampal sclerosis. Epilepsia. 2005;46:939-43.

24. Pohlmann-Eden B, Gass A, Peters CAN et al. Evolution of MRI changes and development of bilateral hippocampal sclerosis during long lasting generalised status epilepticus. J Neurol Neurosurg Psychiatry. 2004;75:898-900.

25. Baxendale S, Heaney D, Thompson PJ et al. Cognitive consequences of childhood-onset temporal lobe epilepsy across the adult lifespan. Neurology. 2010;75:705-11.

26. Arruda F, Cendes F, Andermann F et al. Mesial atrophy and outcome after amygdalohippocampectomy or temporal lobe removal. Ann Neurol. 1996;40:446-50.

27. Sirven JI, Malamut BL, Liporace JD et al. Outcome after temporal lobectomy in bilateral temporal lobe epilepsy. Ann Neurol. 1997;42:873-8.

28. Vogt VL, Witt JA, Malter MP et al. Neuropsychological outcome after epilepsy surgery in patients with bilateral Ammon's horn sclerosis. J Neurosurg. 2014;121:1247-56.

29. King-Stephens D, Mirro E, Weber PB, et al. Lateralization of mesial temporal lobe epilepsy with chronic ambulatory electrocorticography. Epilepsia. 2015;56:959-67.

30. DiLorenzo DJ, Mangubat EZ, Rossi MA et al. Chronic unlimited recording 
electrocorticography-guided resective epilepsy surgery: technology-enabled enhanced fidelity in seizure focus localization with improved surgical efficacy. J Neurosurg.

2014;120:1402-14.

31. Thom M, Liu JYW, Thompson P et al. Neurofibrillary tangle pathology and Braak staging in chronic epilepsy in relation to traumatic brain injury and hippocampal sclerosis: A postmortem study. Brain. 2011;134:2969-81.

Figure legend:

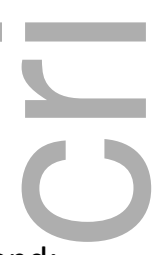

?

Figure 1 - Etiology of bilateral HS. The causes of hippocampal sclerosis were identified by referring centers. In almost two thirds of patients, no clear cause was identified. Infective etiologies and febrile seizures were the main attributable causes with small numbers of patients developing bilateral HS following birth injury, brain trauma, putative antibody-mediated disease, new onset refractory status epilepticus (NORSE) or febrile infection-related epilepsy syndrome (FIRES).

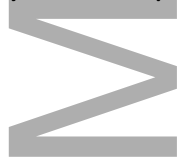

Figure 2: Anti-epileptic medications taken by patients with bilateral HS. The cohort of patients studied had severe drug resistant epilepsy having tried an average of 5.32 medications previously (A) and the mean number of currently taken medications being 2.99 (B). A wide variety of medications had been trialled, predominantly those recommended for treatment of focal epilepsy. Abbreviations: ACET (acetazolamide); CBZ (carbamazepine); CLOB (clobazam); CLOR (clorazepate), CLNZ (clonazepam); DIAZ (diazepam); ESLI (eslicarbazepine); ETX (ethosuximide); FBM (felbamate); GPN (gabapentin); IVIG (intravenous immunoglobulin), LAC (lacosamide); LEV (levetiracetam); LRZ (lorazepam); LTG (lamotrigine); MID (midazolam); OXC (oxcarbazepine); PER (perampanel); PB (phenobarbital), PGB (pregabalin); PHT (phenytoin), PRIM (primidone); RET (retigabine); RUF (rufinamide); TIAG (tiagabine); TOP (topiramate); VPA (valproate); VIG (vigabatrin); ZNS(zonisamide). PWE (persons with epilepsy)

Figure 3: Seizure types in persons with bilateral HS. Focal impaired awareness seizures (FIAS) were the most common seizure type and could be seen in combination with focal aware seizures (FAS) 
and focal to bilateral tonic-clonic seizures (FBTC). However, FAS alone were not observed in any patient with bilateral HS

4: Overall outcomes in patients with bilateral HS. The majority of patients with bilateral HS persisted with medical treatment alone. Eleven patients had resective surgery with either anterior temporal lobectomy (ATL), selective amygdalohippocampectomy (SAH), or corticectomy; 4 underwent prior intracranial EEG monitoring (ICEEG) and 7 patients had single stage resections. Seven underwent ICEEG without subsequent resection. Sixteen patients had undergone, or were awaiting, neurostimulation. Four (4.2\%) became seizure-free on medication alone.

Supporting Figure 1: Comorbidity in patients with bilateral HS. Cognitive (A) and psychiatric (B) comorbidities occurred commonly in persons with bilateral HS. Twenty-one patients had intellectual disability, and 17 had memory impairment. The most common psychiatric comorbidity was depression with or without anxiety. A small percentage of patients experienced psychosis and two patients had abused recreational substances

Supporting figure 2: Ictal and interictal findings in bilateral HS. A wide variety of inter-ictal (A) and ictal (B) features were identified on EEG recording in patients with bilateral HS. Of the cases where records were available, bilateral epileptiform abnormalities were the most common finding.

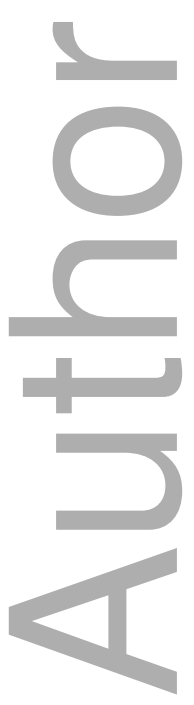

This article is protected by copyright. All rights reserved 
Table 1: Comparison of clinical characteristics between unilateral HS $(n=201)$ vs bilateral HS cases $(n=96)$.

\begin{tabular}{|c|c|c|c|}
\hline r & $\begin{array}{l}\text { Unilateral HS } \\
\qquad(n=201)\end{array}$ & $\begin{array}{c}\text { Bilateral HS } \\
\quad(n=96)\end{array}$ & $P$ value \\
\hline $\begin{array}{l}\text { History of febrile seizures } \\
\text { Yes } \\
\text { No } \\
\text { No data }\end{array}$ & $\begin{array}{l}89(44.3 \%) \\
81(40.3 \%) \\
31(15.4 \%)\end{array}$ & $\begin{array}{c}24(25 \%) \\
63(65.6 \%) \\
9(9.4 \%)\end{array}$ & 0.000236 \\
\hline $\begin{array}{l}\text { History of status epilepticus } \\
\text { Yes } \\
\text { No } \\
\text { No data }\end{array}$ & $\begin{array}{c}23(11.4 \%) \\
69(34.3 \%) \\
109(54.2 \%)\end{array}$ & $\begin{array}{c}26(27.1 \%) \\
64(66.7 \%) \\
6(6.2 \%)\end{array}$ & $<0.00001$ \\
\hline $\begin{array}{l}\text { Focal aware seizures* } \\
\text { Yes } \\
\text { No } \\
\text { No data }\end{array}$ & $\begin{array}{c}84(41.8 \%) \\
106(52.7 \%) \\
11(5.5 \%)\end{array}$ & $\begin{array}{c}21(21.9 \%) \\
71(73.9 \%) \\
4(4.2 \%)\end{array}$ & 0.002011 \\
\hline $\begin{array}{l}\text { Focal impaired awareness seizures } \\
\text { Yes } \\
\text { No } \\
\text { No data }\end{array}$ & $\begin{array}{c}191(95 \%) \\
6(3 \%) \\
4(2 \%)\end{array}$ & $\begin{array}{c}88(91.7 \%) \\
4(4.2 \%) \\
4(4.2 \%)\end{array}$ & 0.474705 \\
\hline $\begin{array}{l}\text { Focal to bilateral tonic-clonic seizures } \\
\text { Yes } \\
\text { No } \\
\text { No data }\end{array}$ & $\begin{array}{c}131(66.7 \%) \\
59(29.4 \%) \\
8(4 \%)\end{array}$ & $\begin{array}{c}64(66.7 \%) \\
28(29.2 \%) \\
4(4.2 \%)\end{array}$ & 0.993164 \\
\hline
\end{tabular}




\begin{tabular}{|c|c|c|c|c|c|c|}
\hline Publication & Phenotype(s) & $\begin{array}{l}\text { Mean duration of } \\
\text { epilepsy, years } \\
\text { (range) }\end{array}$ & History of FC & History of SE or FSE & $\begin{array}{c}\text { History of } \\
\text { encephalitis }\end{array}$ & Other \\
\hline Ravat et al. $^{8}$ & $\begin{array}{l}35 \text { patients with } \\
\text { bHS }\end{array}$ & $15.7(2-47)$ & $\begin{array}{l}19(54.3 \%) \text { history of } \\
\text { FC }\end{array}$ & & $\begin{array}{l}4(11.4 \%) \text { with history } \\
\text { of encephalitic illness }\end{array}$ & \\
\hline Malter et al. ${ }^{9}$ & $\begin{array}{l}\text { Total of } 322 \\
\text { patients: } 31 \text { with } \\
\text { bHS, } 291 \text { with uHS }\end{array}$ & $\begin{array}{l}11 \text { (2-55) with bHS } \\
\text { and } 22(0-65) \text { with } \\
\text { uHS ( } p=0.08, \text { Fisher's } \\
\text { exact test) }\end{array}$ & $\begin{array}{l}8(26 \%) \text { with bHS and } \\
69(24 \%) \text { with uHS had } \\
\text { FC ( } p=0.024 \text {, Fisher's } \\
\text { exact test) }\end{array}$ & $\begin{array}{l}3(9.5 \%) \text { of bHS and } 1(0.3 \%) \text { of } \\
\text { uHS had SE ( } p<0.001, \text { Fisher's } \\
\text { exact test) }\end{array}$ & $\begin{array}{l}8(26 \%) \text { with bHS and } \\
57(20 \%) \text { with uHS } \\
\text { had encephalitis } \\
\text { ( } p=0.44 \text {, Fisher's exact } \\
\text { test) }\end{array}$ & $\begin{array}{l}3 \text { (9.5\%) with } \\
\text { bHS and } 9(3 \%) \\
\text { with uHS had } \\
\text { history of } \\
\text { infantile brain } \\
\text { damage ( } \mathrm{p}=0.07 \text {, } \\
\text { Fisher's exact } \\
\text { test) }\end{array}$ \\
\hline $\begin{array}{l}\text { Van Paesschen et } \\
\text { al. }{ }^{10}\end{array}$ & $\begin{array}{l}\text { Total of } 100 \\
\text { patients: } 7 \text { with } \\
\text { bHS, } 41 \text { with uHS, } 5 \\
\text { with anterior } \\
\text { hippocampal } \\
\text { atrophy, } 47 \text { with } \\
\text { other findings }\end{array}$ & $\begin{array}{l}28 \text { (1-36) with bHS, } \\
20 \text { (1-42) with uHS, } \\
15 \text { (10-17) with } \\
\text { anterior hippocampal } \\
\text { atrophy (not } \\
\text { significant) }\end{array}$ & $\begin{array}{l}0 \text { (0\%) with bHS, } 21 \\
(50 \%) \text { with uHS, and } 4 \\
(80 \%) \text { with anterior } \\
\text { hippocampal atrophy } \\
\text { had FC ( } \mathrm{p}=0.00002 \text {, } \\
\text { Fisher's exact test) }\end{array}$ & & $\begin{array}{l}3(43 \%) \text { with bHS, } 5 \\
(12 \%) \text { with uHS, and } 0 \\
(0 \%) \text { with anterior } \\
\text { hippocampal atrophy } \\
\text { had encephalitis (not } \\
\text { significant) }\end{array}$ & \\
\hline Vanli-Yavuz et & $\begin{array}{l}\text { Total of } 124 \\
\text { patients: } 31 \text { with } \\
\text { bHS, } 93 \text { with uHS }\end{array}$ & & $\begin{array}{l}20 \text { (64.5\%) with bHS } \\
\text { and } 60 \text { (64.5\%) with } \\
\text { uHS had FC (not } \\
\text { significant) }\end{array}$ & $\begin{array}{l}10 \text { with bHS }(32.3 \%) \text { and } 9 \\
(9.7 \%) \text { of uHS had history of SE } \\
(p=0.007, \text { Fisher's exact test) }\end{array}$ & & $\begin{array}{l}15(48.4 \%) \text { of } \\
\text { bHS and } 6 \\
(6.5 \%) \text { with uHS } \\
\text { had mental }\end{array}$ \\
\hline
\end{tabular}

This article is protected by copyright. All rights reserved 


\begin{tabular}{|c|c|c|c|c|c|c|}
\hline 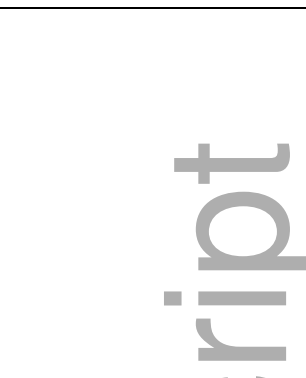 & & & & $\begin{array}{l}10 \text { with bHS }(32.3 \%) \text { and } 9 \\
(9.7 \%) \text { with uHS had history of } \\
\text { FSE with FSE ( } p=0.002 \text {, Fisher's } \\
\text { exact test) } \\
\text { *3 patients had history of both } \\
\text { SE and FSE }\end{array}$ & & $\begin{array}{l}\text { retardation } \\
(p<0.001, \\
\text { Pearson chi- } \\
\text { square) }\end{array}$ \\
\hline Current series & $\begin{array}{l}96 \text { patients with } \\
\text { bHS }\end{array}$ & $30.4(1-79)$ & $\begin{array}{l}24(25.0 \%) \text { with history } \\
\text { of FC }\end{array}$ & 26 (27.0\%) with history of SE & $\begin{array}{l}10(10.4 \%) \text { with } \\
\text { history of } \\
\text { meningoencephalitis }\end{array}$ & $\begin{array}{l}23(24 \%) \text { with } \\
\text { known diagnosis } \\
\text { of intellectual } \\
\text { disability }\end{array}$ \\
\hline
\end{tabular}

Table 2: Comparison of large case series of bilateral HS

bHS: bilateral hippocampal sclerosis; uHS: unilateral hippocampal sclerosis; FC: febrile convulsion; SE: status epilepticus; FSE: febrile status epilepticus

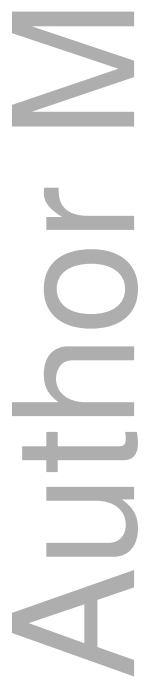

This article is protected by copyright. All rights reserved 


\section{Etiology of bilateral HS}

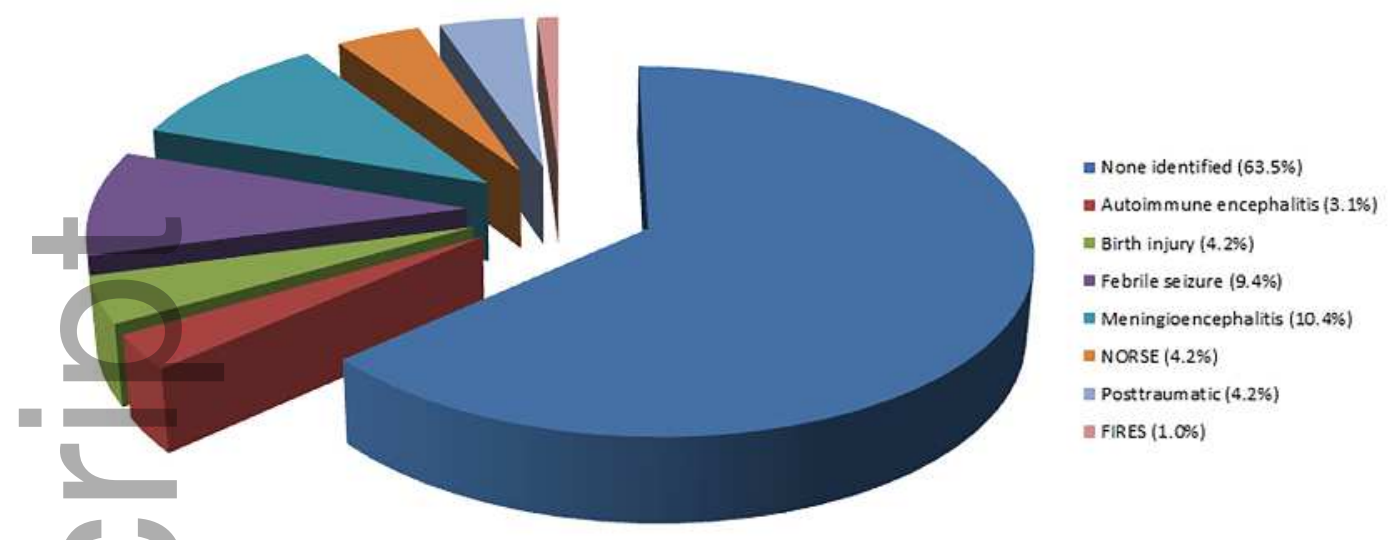

epi_14436_f1.tif

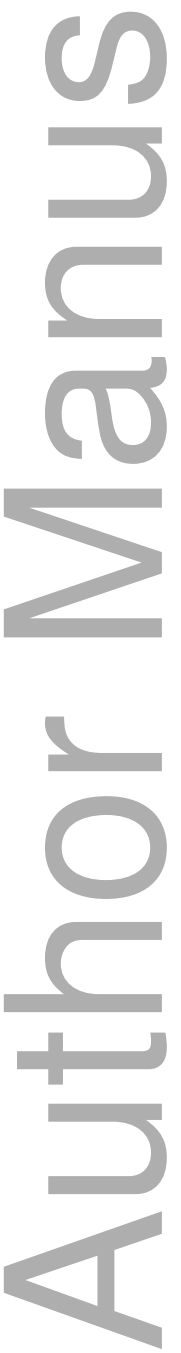




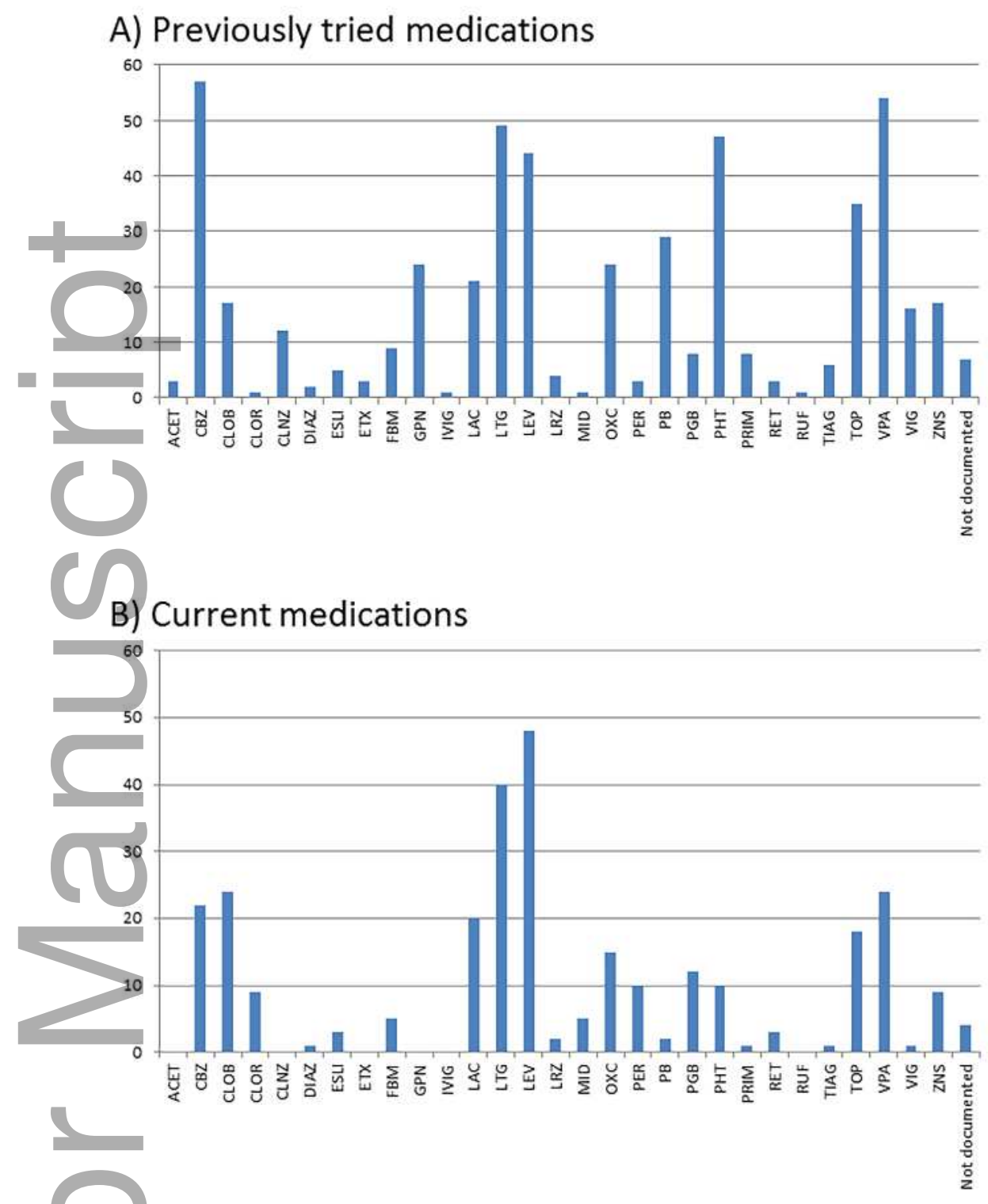

epi_14436_f2.tif 


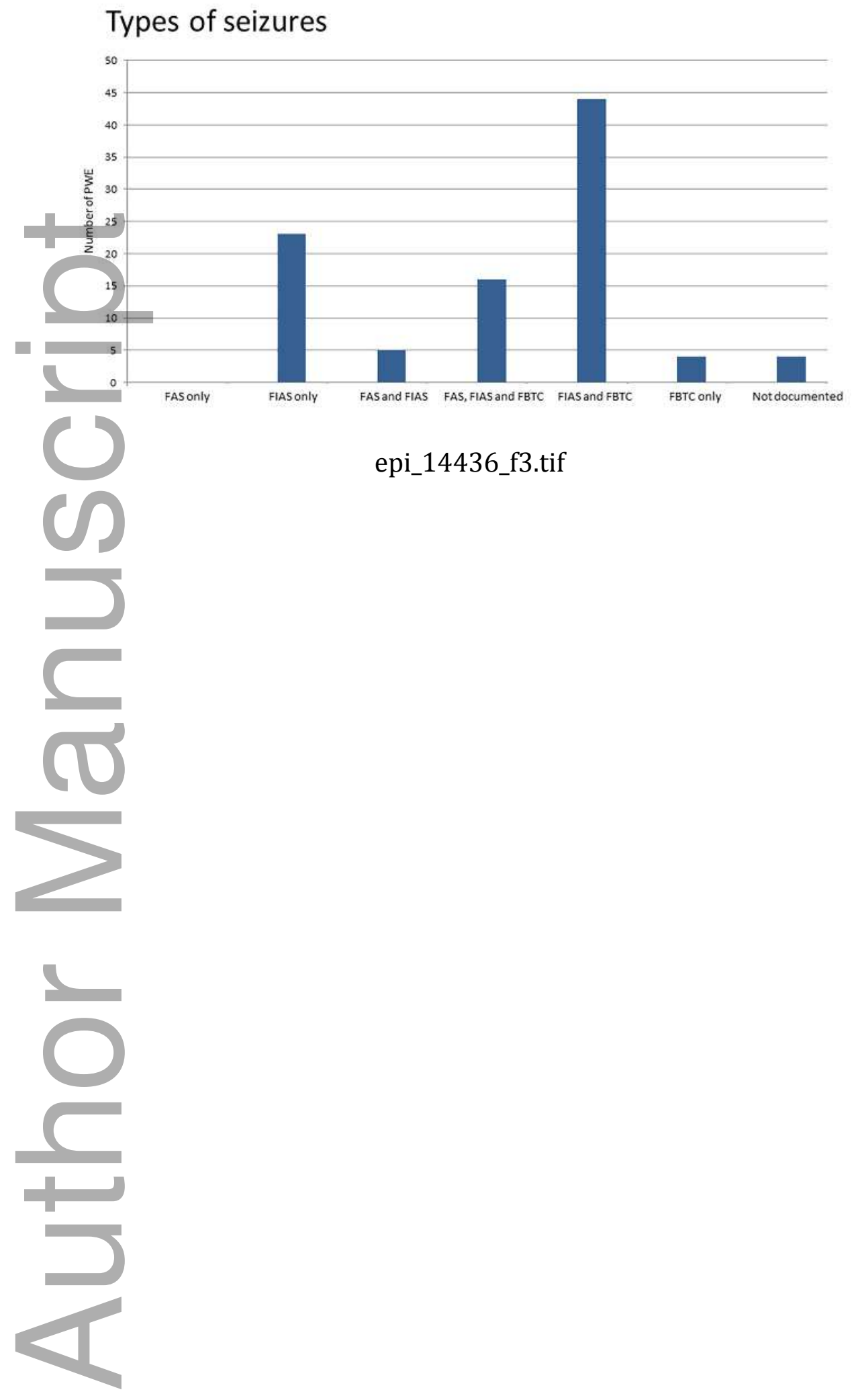

This article is protected by copyright. All rights reserved 


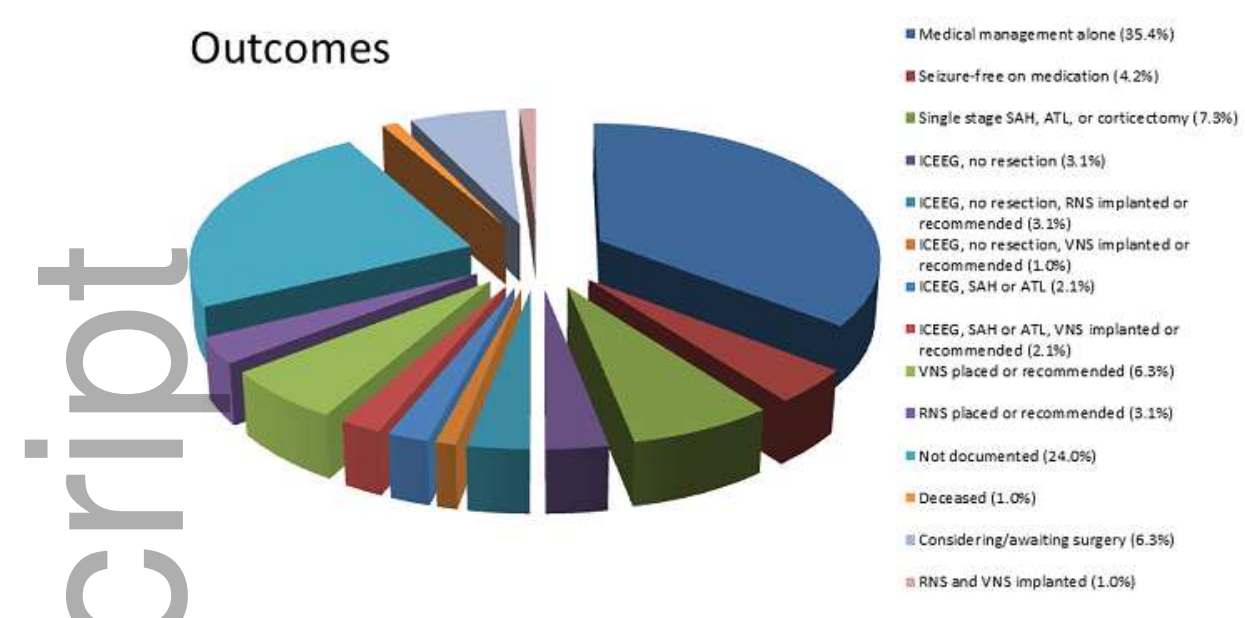

epi_14436_f4.tif

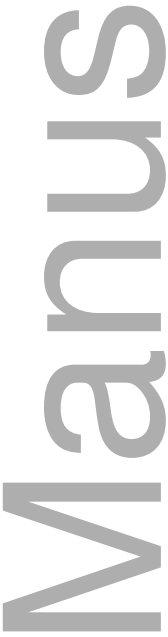

\author{
epi_14436_f4.tif
}

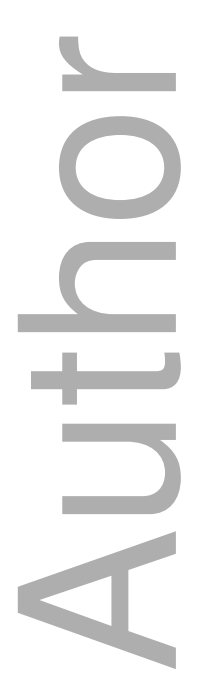




\section{University Library}

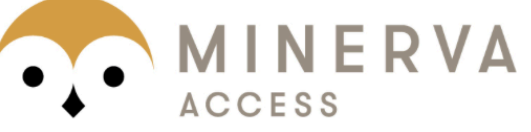

A gateway to Melbourne's research publications

Minerva Access is the Institutional Repository of The University of Melbourne

Author/s:

Sen, A;Dugan, P;Perucca, P;Costello, D;Choi, H;Bazil, C;Radtke, R;Andrade, D;Depondt, C;Heavin, S;Adcock, J;Pickrell, WO;McGinty, R;Nascimento, F;Smith, P;Rees, MI;Kwan, P;O'Brien, TJ;Goldstein, D;Delanty, N

Title:

The phenotype of bilateral hippocampal sclerosis and its management in "real life" clinical settings

Date:

2018-07-01

Citation:

Sen, A., Dugan, P., Perucca, P., Costello, D., Choi, H., Bazil, C., Radtke, R., Andrade, D., Depondt, C., Heavin, S., Adcock, J., Pickrell, W. O., McGinty, R., Nascimento, F., Smith, P., Rees, M. I., Kwan, P., O'Brien, T. J., Goldstein, D. \& Delanty, N. (2018). The phenotype of bilateral hippocampal sclerosis and its management in "real life" clinical settings. EPILEPSIA, 59 (7), pp.1410-1420. https://doi.org/10.1111/epi.14436.

Persistent Link:

http://hdl.handle.net/11343/284100 